\title{
¿POR QUÉ INVESTIGAR ASPECTOS POSITIVOS EN LA VEJEZ?. CONTRIBUCIONES PARA UN CAMBIO DE PARADIGMA
}

\author{
POR QUE INVESTIGAR ASPECTOS POSITIVOS NA \\ VELHICE? CONTRIBUIÇÕES PARA UMA MUDANÇA \\ DE PARADIGMA
}

\author{
Claudia J. Arias ${ }^{*}$ \\ Ricardo Iacub ${ }^{* *}$
}

\begin{abstract}
RESUMEN
Este trabajo se centrará en reflexionar acerca de los motivos por los cuales consideramos importante investigar aspectos positivos en la vejez. Para ello, en primer lugar analizaremos resultados de variados trabajos de investigación que han explorado cuestiones como el bienestar, el capital psíquico, las relaciones de apoyo social, las experiencias de fluidez y la sexualidad en los adultos mayores. Estos nos permitirán problematizar y cuestionar ciertos presupuestos acerca de la vejez. Luego intentaremos repensar diversos aspectos referidos a los métodos que implementamos en nuestras investigaciones en el campo de la Gerontología. Esto implicará efectuar un análisis crítico que incluya distintos niveles de orden metodológico tales como los problemas que nos planteamos, los diseños que proponemos, los instrumentos con los que exploramos, las poblaciones y muestras que seleccionamos y el tipo de conocimientos que alcanzamos. Finalmente, se efectuarán propuestas orientadas a generar cambios en el paradigma vigente. Se apunta a contemplar, tanto a nivel teórico como metodológico, la presencia de los aspectos positivos y negativos presentes en el proceso de envejecimiento. De este modo se pretende lograr una mayor comprensión de su complejidad así como captar los variados matices que éste adquiere como resultado de la relación dialéctica entre unos y otros.
\end{abstract}

Palabras clave: Adulto Mayor. Envejecimiento. Paradigma de la vejez. Aspectos positivos de la vejez.

\section{RESUMO}

Este trabalho centra-se em refletir acerva dos motivos pelos quais consideramos importante investigar aspectos positivos na velhice. Para isto, em primeiro ligar analisaremos resultado de vários trabalhos de investigação que tem explorado questões como bem estar, o capital psíquico, as relações de apoio social, as experiências de fluidez e a sexualidade dos adultos maiores. Estes nos permitirão problematizar e questionar certos pressupostos acerca da velhice. Logo tentaremos repensar diversos aspectos referidos aos métodos que implementamos em nossas

\footnotetext{
“Magister en Psicología Social. Docente de grado y posgrado. Investigadora y Directora de proyectos. Coordinadora de Talleres de Extensión para adultos mayores. Facultad de Psicología. Universidad Nacional de Mar del Plata.

" Doutor em Psicologia e Especialista em Mediana Edad. Prof. e investigador de la Universidad de Buenos Aires.
} 
investigações no campo da Gerontologia. Isto implicará efetuar uma análise crítica que inclua distintos níveis de ordem metodológica tais como consideramos os problemas, os desenhos que propomos, os instrumentos que exploramos, as populações e amostras que selecionamos e o tipo de conhecimento que alcançamos. Finalmente, se efetuarão propostas orientadas a gerar mudanças no paradigma vigente. Ele aponta para contemplar, tanto a nível teórico como metodológico, a presença dos aspectos positivos e negativos presentes no processo de envelhecimento. Deste modo se pretende alcançar uma maior compreensão de sua complexidade assim como captar os variados refinamentos que este adquire como resultado da relação dialética entre uns e outros.

Palavras-chave: Velhice. Paradigma da velhice. Aspectos positivos da velhice.

\section{Introducción}

Durante décadas la Psicología priorizó la investigación de aspectos negativos como el malestar, la depresión y la infelicidad más que la comprensión, potenciamiento o estimulación de los aspectos positivos del ser humano. De un modo similar, aunque aún más exacerbado, los estudios acerca de la vejez resaltaron el deterioro y lo patológico contribuyendo a consolidar narrativas tanto científicas como sociales que sostienen una visión negativa de esta etapa vital. Es así que la vejez y el envejecimiento han sido históricamente estudiados desde una perspectiva que ha enfatizado los aspectos biológicos, centrándose en las pérdidas y déficits y cuyos hallazgos han desconocido la amplia variabilidad existente entre las personas. En definitiva las generalizaciones que se han planteado y difundido acerca de la vejez sostienen y proponen un único destino posible para esta etapa de la vida. Destino que, por común e ineludible, pareciera resistirse a las diferencias individuales, a las variadas condiciones de vida, así como a las trayectorias y elecciones personales que el sujeto ha hecho a lo largo de su vida y que continúa haciendo en la vejez. Aunque en la actualidad la homogeneidad del proceso de envejecimiento resulte evidentemente falsa, ésta continúa sosteniéndose en variados estereotipos negativos que aún contribuyen a mantener vigente dicha visión deficitaria de la vejez.

Sin embargo, en las últimas décadas, los estudios que se han orientado al estudio de aspectos positivos han aportado hallazgos de gran importancia acerca de esta etapa vital. Cada vez más se está destacando la relevancia de incluir el estudio de los aspectos positivos y la necesidad de avanzar en el conocimiento y profundización de los mismos.

\section{¿Qué entendemos por aspectos negativos y positivos?}

Estos términos nos permiten diferenciar entre los aspectos que involucran malestar, sufrimiento, patología, déficits, por un lado y aquellos que incluyen recursos, habilidades y capacidades que propician el desarrollo y el potencial humano por otro. Los adjetivos positivo y negativo de ningún modo se utilizan con un fin valorativo para diferenciar entre lo bueno y lo malo, ni como polos opuestos que pueden presentarse en forma pura. Por una parte, los juicios morales no corresponden al campo de la ciencia y por otra, como dijimos anteriormente, dichos aspectos no pueden pensarse individualmente, sino en un permanente interjuego. El hecho de incorporar el estudio de aspectos positivos en esta etapa de la vida no implica proponer un modelo ideal de vejez en el que solo estos estén presentes. Dicha propuesta, además de imposible e inalcanzable, caería nuevamente en el error de suponer una homogeneidad del proceso de envejecimiento que criticamos al paradigma deficitario. Si bien en una variante opuesta, que en lugar de las pérdidas pregonara exclusivamente 
las ganancias, nuevamente estaríamos proponiendo una vejez uniforme que desconoce las diferencias individuales.

Por el contrario, ambos aspectos - positivos y negativos- se conjugan a lo largo de toda nuestra vida en una necesaria complementariedad. Como han resaltado diversos autores de la Psicología Positiva no se trata de corrernos de un modo radical desde un extremo hacia el otro negando el permanente interjuego entre ambos. Para hacer referencia a la relación dinámica existente entre los aspectos positivos y negativos, Ryff y Singer (2007) analizan las "ironías o las paradojas del bienestar" demostrando que el potencial humano se desarrolla al enfrentar situaciones adversas y dificultades. En este sentido, la resiliencia es un concepto que permitió entender y dar cuenta de este tipo de fenómenos. Además, plantean el interjuego entre estos aspectos al afirmar que tanto las emociones positivas como las negativas caracterizan a nuestros más profundos y significativos lazos humanos" $\mathrm{y}$ al proponer que "la ausencia de emociones o experiencias negativas no es lo que define una vida buena y rica en experiencias, sino cómo se manejan esos desafíos y las dificultades, cómo se responde a ellos y cómo se tratan y transforman" (Ryff \& Singer, 2007: 378).

Los fenómenos psicológicos deben comprenderse de un modo global, considerando que en todas las etapas de la vida se encuentran fortalezas y debilidades y que todos los cambios generan tanto pérdidas como ganancias (Baltes \& Freund, 2007).

Durante los últimos 10 años, desde la Psicología Positiva se están realizando importantes aportes, ampliando el foco desde la preocupación casi exclusiva por reparar el daño, hacia un énfasis en el fortalecimiento de las cualidades positivas y el desarrollo de las potencialidades humanas (Seligman, 1998). Asimismo se está avanzando en la comprensión de las condiciones, procesos y mecanismos que llevan a estados subjetivos, sociales y culturales que caracterizan una buena vida (Seligman, 1998, 2003; Seligman \& Csikszentmihalyi, 2002; Gancedo, 2008) y favorecen el bienestar. En este sentido se ha enfatizado la necesidad de complementar el abordaje de los aspectos psicopatológicos con el de las capacidades y cualidades que poseen los individuos en el contexto en que viven. Esta complementariedad permitiría una mayor comprensión del espectro de experiencias que definen y sostienen el malestar y el sufrimiento, así como de las posibilidades de afrontarlo, resolverlo y superarlo o prevenirlo. Interesa fundamentalmente comprender y propiciar la felicidad y el bienestar, así como la realización plena del potencial humano.

En los estudios actuales, desde la Gerontología y la Psicología de la Vejez, se está incluyendo el estudio de los recursos, fortalezas, habilidades y competencias, efectuando aportes que permiten una mayor comprensión de la variabilidad y complejidad del proceso de envejecimiento humano. Sin embargo, su investigación sistemática y la exploración de las variables que intervienen en este logro, data solo de aproximadamente unas cuatro décadas.

\section{¿Qué nos ha aportado la investigación de aspectos positivos en la vejez?}

Una de las mayores contribuciones que han realizado las investigaciones recientes dirigidas al estudio de aspectos positivos en la vejez ha sido el cuestionamiento reiterado y fundamentado de una amplia variedad de falsos supuestos acerca de esta etapa vital. En este sentido, diversos trabajos han mostrado que los adultos mayores pueden ser felices, disponer de recursos de apoyo social suficientes, disfrutar de su sexualidad, sentir elevados niveles de bienestar, estar satisfechos con sus vidas y poseer múltiples fortalezas personales, entre otros aspectos positivos.

A continuación analizaremos detalladamente algunos resultados de investigaciones que han abordado estas temáticas demostrando que variados prejuicios y estereotipos negativos acerca de la vejez, aunque gocen de amplio consenso, carecen de fundamentación científica e impiden visibilizar las heterogéneas formas de envejecer.

\section{Bienestar psicológico, Satisfacción vital y Felicidad en la vejez}

Los estudios acerca del bienestar en la vejez se han multiplicado en las últimas décadas, convirtiéndose en el aspecto positivo que ha sido más extensamente explorado (Fernández Ballesteros, 
2007). Estas investigaciones han avanzado en la comprensión de los aspectos asociados al mismo. En este sentido se han identificado las relaciones entre el bienestar psicológico y variables tales como la autoestima (Izal \& Montorio, 1993), la actividad social (Okun, Stock, Haring \& Witter, 1984), el afrontamiento (Vera Noriega, Sotelo Quiñones \& Domínguez Guedea, 2005), los rasgos de personalidad (Chico Librán, 2006), el apoyo social (Arias, 2004; Muchinick, 1984), el funcionamiento del self (Liberalesso Neri, 2002), el nivel y estilo de vida (García-Viniegras \& González-Benítez 2000), variables sociodemográficas, el estado funcional, la salud (Izal \& Montorio, 1993) y la religión (Levin \& Chatters, 1998). Si bien estos hallazgos han confirmado múltiples aspectos que contribuyen a mejorar o a deteriorar el bienestar en la vejez, no han aportado evidencia a favor de su disminución con la edad. Por el contrario, los estudios han demostrado que el bienestar subjetivo como medida global no experimenta cambios con el paso del tiempo. Si bien pueden presentarse modificaciones en algunas dimensiones, estas son relativamente poco importantes (Villar, Triado, Solé \& Osuna, 2003).

Un trabajo realizado en Mar del Plata mostró que la distribución de puntajes de bienestar global de los adultos mayores que viven en hogares particulares no presentaba diferencias significativas con la correspondiente a la población adulta (Arias \& Soliverez, 2009a, 2009b). Los adultos mayores también han informado niveles elevados de satisfacción vital mostrando congruencia entre los objetivos propuestos y los alcanzados y el logro de un positivo balance de vida (Arias, 2004). Las investigaciones actuales que han explorado la felicidad en la vejez también han obtenido resultados en la misma dirección, mostrando que los adultos mayores no solo informan niveles de felicidad similares o superiores a los adultos jóvenes o de la mediana edad (Lacey, Smith \& Ubel, 2006; Wood, Kisley \& Burrows, 2007) sino que también poseen un mayor control de sus emociones y menores niveles de afecto negativo (Carstensen, Pasupathi, Mayr \& Nesselroade, 2000). La denominada "paradoja de la vejez" radica en que a pesar de que existe cierto declive físico y cognitivo, se mantiene e incluso se incrementa el bienestar psicológico (Carstensen \& Charles, 1998).
En síntesis, estos resultados de investigaciones muestran que el bienestar, la felicidad, la satisfacción vital y el afecto positivo no decrecen en la vejez. Al igual que en otras etapas de la vida, estos pueden verse afectados por ciertas circunstancias particulares y por la significación que el sujeto les otorgue. De esta manera se pueden superar prejuicios que pensaban a los adultos mayores como sujetos depresivos o de humor negativo (Arias, Iacub, Soliverez y cols., 2008).

\section{El Capital psíquico y su incremento en la vejez}

Las fortalezas y virtudes humanas han sido consideradas aspectos positivos de gran relevancia y están siendo valoradas en la actualidad como factores que inciden en el bienestar, posibilitando un afrontamiento más saludable de las situaciones de crisis y de estrés (Seligman, Steen, Park \& Peterson, 2005). Si bien la investigación acerca de estas temáticas está siendo ampliamente desarrollada, específicamente el capital psíquico - entendido como el conjunto de factores y procesos que permiten a un sujeto aprender a protegerse, a sobrevivir y a generar fortalezas personales (Casullo, 2006)- constituye un constructo de reciente conceptualización y operativización, razón por la cual su estudio está en una etapa incipiente. En nuestro país son aún escasas las investigaciones de carácter empírico y técnico sobre este tema, y más aún si se orientan a su evaluación en los adultos mayores. La mayoría de los hallazgos alcanzados se centran fundamentalmente en la población adolescente y adulta joven (Casullo y cols, 2002; Posada, Castañeiras \& Arias, 2008a, 2008c). En lo referido a los adultos mayores, los estudios se han focalizado en el estudio específico de algunas dimensiones como la sabiduría (Baltes, 2003; Ardelt, 2003), la espiritualidad (Rivera Ledesma \& Montero López, 2007; Yoffe, 2008; Frazier, Mintz \& Mobley, 2005), la capacidad de desarrollar proyectos nuevos (Lawton, Moss, Winter \& Hoffman, 2002), el sentido del humor (Ryff, 1989), entre otras. La reciente exploración del capital psíquico de manera más global y en este grupo etáreo ha mostrado que los adultos mayores disponen de elevados y variados recursos de tipo cognitivo, afectivo y psicosocial (Arias, Castañeiras 
\& Posada, 2008; Posada, Castañeiras, Arias, 2008b). En una investigación muy amplia realizada en la ciudad de Mar del Plata se trabajó con una muestra conformada por 900 adultos de ambos sexos pertenecientes a cinco grupos de edad: 16 a 26 años, 27 a 37 años, 38 a 48 años, 49 a 59 años y 60 a 75 añosa la que se le administró la Escala CAPPSI (Casullo, 2006) que evalúa cinco dimensiones del capital psíquico 1) las capacidades cognitivas para adquirir y usar formas de conocimiento, 2) las capacidades emocionales para desarrollar proyectos, superando obstáculos, 3) las habilidades cívicas para lograr mejor participación ciudadana, 4) las capacidades para establecer vínculos interpersonales y 5) el sistema de valores como metas que orientan los comportamientos. Los resultados muestran que las puntuaciones promedios correspondientes a las cinco dimensiones aumentaban al incrementarse la edad, correspondiendo en todos los casos los puntajes más altos al grupo de 60 a 75 años. Excepto en la dimensión correspondiente a capacidades cognitivas, en las cuatro restantes, estas diferencias favorables a los adultos mayores fueron estadísticamente significativas (Arias, Posada \& Castañeiras, 2009).

En un estudio posterior se profundizó el análisis a fin de identificar las fortalezas que se hallaban presentes en los adultos mayores en mayor medida que en los adultos jóvenes. Entre ellas se destacaron: la Motivación para aprender cosas nuevas, la Sabiduría, la Curiosidad, la Honestidad, la Autoestima, la Persistencia, la Inteligencia emocional, el Amor, el Sentido del humor, la Empatía y el Altruismo, el Sentido de justicia, la Capacidad para perdonar, la Gratitud y la Espiritualidad (Arias, Posada \& Castañeiras, 2009). Posteriormente, a fin de conocer si este incremento de los aspectos involucrados en el capital psíquico continuaba aún en el grupo de los adultos mayores de más edad se desarrolló una investigación que exploraba el capital psíquico en personas de 75 a 91 años. En este caso se confirmó su estabilidad hasta las edades más avanzadas e incluso su incremento en la dimensión correspondiente a los valores que orientan nuestros comportamientos y en los aspectos referidos a sabiduría, amor y capacidad para perdonar (Arias, 2009).

Los hallazgos de estos trabajos muestran que las virtudes y fortalezas se profundizan en la vejez y que el desarrollo personal continúa hasta las edades más avanzadas. De este modo aportan nueva evidencia en el cuestionamiento de la vejez como sinónimo de pérdida y deterioro.

\section{Presencia de Experiencias de Fluidez en los adultos mayores}

Las experiencias de fluidez constituyen emociones positivas cuya exploración tiene un gran interés en la vejez. La profundización del conocimiento del que disponemos en la actualidad y la investigación acerca de las emociones con las que estas experiencias se relacionan resultan fundamentales ya que inciden en el bienestar y en la calidad de vida (Csikszentmihalyi \& Csikszentmihalyi, 1998). Las experiencias de fluidez hacen referencia a un especial estado psicológico que pueden experimentar las personas en la ejecución de actividades motivadas intrínsecamente. La activación motivacional depende en gran medida de la correspondencia entre las habilidades requeridas para la ejecución de la tarea y las que el sujeto posee. Además la fluidez se caracteriza por la formulación clara de las metas, así como por una distorsión en la percepción del tiempo y un olvido de los problemas. Este estado ha sido considerado como "óptimo" por el alto grado de concentración e interés, la implicación profunda, la motivación elevada $\mathrm{y}$ la sensación de control. También se correlaciona con sensación de satisfacción, afecto positivo e incremento de la autoestima después de realizada la actividad (Carr, 2007).

En una investigación cuyo objetivo consistía en explorar la presencia/ausencia de fluidez en adultos jóvenes y adultos mayores, así como el tipo de actividad que la generaba, pudo observarse que gran parte de los adultos mayores entrevistados presentaban este tipo de experiencias y en proporciones similares a la de los adultos jóvenes (Arias \& Sabatini, 2008; Sabatini \& Arias, 2009).

Con respecto a las actividades que las generan, en ambos grupos de edad aparecieron prácticas deportivas, artísticas e intelectuales. Sólo los adultos jóvenes mencionaron el trabajo y el estudio como origen de dichas experiencias. En los adultos mayores las actividades que generaban estas experiencias se mencionaron: escuchar 
música, pintar, practicar yoga, asistir a espectáculos o conferencias y practicar deportes como pesca, automovilismo, natación y gimnasia. Los adultos mayores coincidieron en considerar que la fluidez se origina por el placer, gusto y agrado que produce la actividad, la concentración, el interés, el dominio que requiere el desarrollo de la misma, el encontrarse a solas y la alineación con uno mismo.

Se observaron diferencias entre ambos grupos etáreos en lo referido al motivo que las origina. Mientras los adultos jóvenes la atribuyen mayoritariamente a la concentración y la creatividad que implica la actividad; los adultos mayores priorizan el placer y el disfrute de la misma (Arias \& Sabatini, 2008; Sabatini \& Arias, 2009).

En síntesis, los estudios que han explorado la fluidez en la vejez permiten concluir dos cuestiones de gran importancia. En primer lugar muestran que, aunque en esta etapa de la vida suelen perderse roles que la generan -como los laborales-, las emociones positivas de fluidez siguen presentes y en proporciones similares a la de los adultos jóvenes. En segundo lugar, se observa que los adultos mayores además de poder experimentar fluidez a partir de actividades que continúan desde etapas anteriores de la vida, incorporan nuevas ocupaciones que también generan dicho estado.

\section{EI Apoyo social en los adultos mayores}

Los recursos de apoyo social han sido ampliamente valorados en la vejez. Las evidencias acerca del efecto positivo de las redes suficientes y funcionales sobre el bienestar biopsicosocial de los adultos mayores han sido muy extensas y variadas. Sin embargo, se han considerado como reglas generales: a) que durante la vejez la red sufría importantes pérdidas, b) que se dificultaba tanto el mantenimiento de antiguos vínculos como la incorporación de nuevas relaciones y c) que, como consecuencia de ello, los adultos mayores disponían de escasos recursos de apoyo social, sufriendo aislamiento y soledad muy frecuentemente, así como patologías derivadas de ello.

Sin embargo, estos supuestos también están siendo cuestionados en la actualidad. Al respecto Carstensen y Charles (2007) sostienen que las explicaciones que se han brindado acerca de la disminución de las relaciones sociales en la vejez se han basado más en ideas y estereotipos -en muchos casos incorrectos- que en hallazgos de investigación.

Los estudios recientes orientados a la evaluación de las redes de apoyo social en los adultos mayores no han confirmado su insuficiencia como un hecho generalizado. Por el contrario han mostrado una muy amplia variabilidad al respecto, pudiendo observarse casos en los que la red reduce su tamaño, se distribuye irregularmente, se homogeneiza y pierde funcionalidad, así como redes de niveles estructurales y funcionales medios y elevados. Si bien algunos hechos como la muerte de familiares y amigos, la migración en la vejez, la jubilación y el ingreso a una residencia pueden impactar negativamente sobre la red, la vejez no implica aislamiento y soledad, ni determina la insuficiencia de los apoyos sociales de un modo inexorable. Por el contrario gran parte de los adultos mayores disponen de recursos de apoyo de niveles de suficiencia y funcionalidad elevados y continúan incorporando nuevos vínculos a su red (Arias, 2004; 2009). Si bien el tamaño de la red tiende a reducirse en la vejez, el número de relaciones íntimas y el apoyo emocional del cual se dispone es relativamente estable a lo largo de la vida y hasta las edades más avanzadas (Due, Holstein, Lund, Modvig y Avlund, 1999; Antonucci, 2001). Seguramente los motivos que, en algunos casos, tienden a generar disrupciones severas de la red durante la vejez no se reduzcan al aumento de la edad y sea necesario incluir otras variables individuales, familiares, culturales y sociales que expliquen esa situación.

En el estudio de las redes en la vejez una vez más se ha enfatizado el estudio de las pérdidas hasta el punto de considerar la insuficiencia de los apoyos sociales como una característica propia de esta etapa vital.

\section{La Sexualidad en la vejez}

La creencia de que la sexualidad no era posible ni deseable en la vejez ha sido ampliamente aceptada hasta épocas recientes. Sin embargo las investigaciones realizadas mostraron que el declive de la sexualidad tiene que ver más con la viudez y el deterioro de la salud que con el aumento de la edad. Por el contrario, estudios longitudinales mostraron 
que los adultos mayores tienen una sexualidad activa y que disfrutan de ella (Ganen, 2002; Janus \& Janus, 1993; Master, Jonson \& Kolodny, 1995).

Iacub (2006) a partir del análisis de variadas creaciones culturales: documentos, libros sagrados, leyes, producciones artísticas, efectúa un proceso de de-construcción que permite entender el origen de estereotipos negativos acerca de la sexualidad en la vejez. En dicho análisis se brindan elementos para repensar mandatos, falsos argumentos y prejuicios que afirman que la sexualidad no es importante en la vejez, que los adultos mayores no tienen deseos ni necesidades sexuales, que el sexo en esta etapa de la vida es perjudicial para su salud y hasta que las manifestaciones sexuales en los adultos mayores son patológicas. La identificación de los supuestos en los que se basan múltiples estereotipos relacionados con el erotismo en la vejez permite comprender sus significados en el relativismo cultural, en el contexto de diversas concepciones y a la luz de mecanismos de poder a partir de las que se generan y sostienen. Desde una perspectiva positiva que se orienta a resaltar las posibilidades en lugar de las limitaciones, Iacub centra el análisis en el erotismo, ya que abre un área más amplia que incluye el deseo, el amor e implica una amplia variedad de formas posibles de lograr placer. El erotismo y la consideración de la sexualidad en un sentido amplio descalifican por completo su imposibilidad durante la vejez y las limitaciones impuestas exclusivamente por la edad.

\section{Acerca de los métodos y diseños que utilizamos...}

En el inicio nos planteamos efectuar una reflexión crítica acerca de las investigaciones que desarrollamos para abordar la vejez y el envejecimiento. Propusimos que esta reflexión involucraría aspectos tales como los métodos y los diseños que implementamos, las poblaciones y muestras con las que trabajamos, los problemas que nos planteamos e incluso los conocimientos que logramos.

En las Ciencias sociales en general debido tanto a la complejidad de los fenómenos estudiados como a la abstracción de los constructos con los que trabajamos y a las dificultades para desarrollar instrumentos que nos permitan efectuar mediciones precisas, el tipo de diseños de investigación que se plantea en general limita las posibilidades de establecer relaciones causales entre las variables. De hecho, si efectuáramos una consulta en bases bibliográficas acerca de las producciones científicas en esta área notaríamos el predominio de estudios que solo buscan establecer correlaciones entre variables $\mathrm{y}$ de ningún modo identificar causas y efectos. Por otra parte, los problemas que investigamos en la mayoría de los casos involucran fenómenos que son multideterminados y por lo tanto para que un efecto se produzca deben conjugarse múltiples factores. Esto nos impide identificar una única variable como la causa exclusiva de un determinado efecto. Por estas razones también se han desarrollado estudios desde metodologías cualitativas que permitan captar en mayor grado la riqueza de este tipo de fenómenos y explorarlos más profundamente. A partir de lo anteriormente expuesto, ¿Podemos pensar que para sentir bienestar, fluidez, disponer de apoyo social y de otros aspectos positivos es suficiente solo con ser joven? o ¿que para sentir malestar o perder todo lo anterior es suficiente con llegar a la vejez?. Se ha demostrado ampliamente que estos constructos son multidimensionales y además plurideterminados por múltiples variables que se combinan entre sí y están fuertemente interrelacionadas. Sin embargo, a pesar de esa complejidad y de las dificultades detalladas, la edad, ha sido considerada la variable independiente por excelencia a la hora de explicar por sí sola múltiples efectos: deterioro cognitivo, pérdida de relaciones sociales, depresión, dependencia, déficits para desarrollar de manera autónoma las actividades de la vida diaria, inactividad sexual, aparición de patologías físicas y psíquicas, etc. Aunque resulta evidente que la edad de manera exclusiva no puede explicar todos estos efectos y que para ello se deben incluir múltiples aspectos de manera conjunta y en una combinación determinada, fueron necesarias reiteradas evidencias para apenas comenzar a poner en duda este fuerte presupuesto.

\section{Hacia un cambio de paradigma}

Las concepciones filosóficas y teóricas que el investigador posee previamente tienen una enorme importancia sobre la investigación que este efectúe. 
Dichas concepciones orientan fuertemente hacia la exploración e investigación de determinadas problemáticas a la vez que nos alejan de otras. De un modo general, en el estudio de la vejez, se han priorizado los aspectos negativos, aportando evidencia empírica a la hipótesis de pérdidas durante esta etapa vital.

Históricamente, la orientación de nuestras búsquedas y la priorización de los aspectos negativos en los estudios acerca de la vejez y el envejecimiento, han condicionado nuestros hallazgos, aportando evidencia exclusivamente sobre estos y contribuyendo a consolidar una percepción negativa de esta etapa vital. Los resultados de investigaciones anteriormente analizadas nos permiten repensar algunas cuestiones que impregnan las representaciones sociales $\mathrm{e}$ incluso el discurso científico acerca de la vejez. Debemos considerar que los estereotipos recaen sobre los individuos y los limitan y condicionan en su modo de comportarse. Los adultos mayores en muchos casos asumen el lugar que socialmente se les asigna ya que es lo que se espera de ellos o lo que se considera normal.

Como hemos analizado anteriormente cuando las investigaciones incluyen también la exploración de aspectos positivos nos encontramos con hallazgos inesperados para un paradigma deficitario, que muestran de manera reiterada que en la vejez también hay ganancias.

Si nuestras creencias y teorías guían desde el inicio el proceso de investigación debemos ser capaces de diseñar estudios novedosos -respecto de los antiguos esquemas- que permitan captar la variabilidad interindividual que caracteriza a la vida humanay que se incrementa enlavejez. Estoinvolucra no solo la inclusión de temáticas escasamente investigadas sino también la exploración del interjuego entre los aspectos positivos y negativos. La inclusión del complemento olvidado posibilitará un conocimiento más completo y profundo del proceso de envejecimiento y la modificación de la visión deficitaria y patológica de la vejez. Por otra parte, el desarrollo de este tipo de investigaciones contribuirá a diseñar intervenciones que incluyan los aspectos positivos en estrategias terapéuticas así como en la prevención y la promoción de la salud.

Al respecto y considerando la amplia heterogeneidad existente en la vejez es necesario estudiar las variadas subpoblaciones de adultos mayores. Además, a fin de evitar sesgos y parcializaciones del conocimiento, dentro de la muestras debemos incluir casos diversos o relativizar cuidadosamente nuestros resultados de acuerdo con los sujetos seleccionados.

Los estudios transversales, que comparaban el desempeño de adultos jóvenes y adultos mayores en un tiempo único y a partir de los cuales se dedujo que el aumento de la edad generaba variadas pérdidas a nivel cognitivo, constituyen claros ejemplos de errores cometidos en la elección del diseño de investigación. Posteriormente la implementación de estudios longitudinales permitió reinterpretar los resultados con la inclusión del denominado efecto de cohorte. Si nuestras investigaciones a partir de diseños específicos logran captar los variados matices que adquiere el proceso de envejecimiento posiblemente veamos más claramente que las regularidades son escasas y que la edad por sí sola pueda explicar muy poco.

La posibilidad de desarrollar nuevas estrategias metodológicas nos permitirá descubrir hallazgos novedosos que nos ayuden a de-construir y problematizar supuestos. El cambio de un paradigma deficitario fuertemente arraigado y presente en variadas narrativas contemporáneas requerirá la generación de conocimientos acerca de los aspectos tanto negativos como positivos y una comprensión más profunda de esta etapa vital. No se propone instalar "lecturas binarias", ni "relatos polarizados" que, al negar viejas definiciones de un modo esquemático, no logran salir de los propios límites del prejuicio (Iacub, 2008). Se apunta a desarrollar investigaciones que permitan a) complejizar el análisis, incluyendo nuevas variables que ayuden a comprender la amplia variabilidad interindividual y los diversos modos de vivir la vejez, b) deconstruir presupuestos fundados en un paradigma deficitario y c) producir narrativas acerca de la vejez y el envejecimiento que, a partir de la inclusión tanto de los aspectos negativos como de los positivos y del análisis de su relación dialéctica (Ryff \& Singer, 2007), eviten las simplificaciones y parcializaciones del conocimiento y permitan enriquecer nuestro saber acerca de esta etapa de la vida (Arias, 2008).

La socialización y difusión de estos hallazgos y la llegada a diversos ámbitos tanto científico- 
académicos como a la comunidad en general será de gran importancia para la modificación de la concepción negativa de la vejez. Si bien los cambios que se han producido en este sentido son incipientes y la consolidación de una visión más positiva demandará tiempo y esfuerzo, actualmente diversas narrativas están empezando a mostrar una imagen diferente de la vejez en la que lo positivo también es posible...

\section{Referencias bibliográficas}

ANTONUCCI, T. C. Social relations: an examination of social networks, social support, and sense of control. En: BIRREN, J. E.; SCHAIE, K. W. Handbook of the psychology of aging. Londres: Academic Press, 2001.

ARDELT, M. Evaluación empírica de una escala de sabiduría tridimensional. Universidad de Florida, 2003. USA Disponible en: <http:www. sagepublications.com>.

ARIAS, C. Red de apoyo social y bienestar psicológico en personas de edad. Mar del Plata: Suárez, 2004.

El modelo teórico del apoyo social. Facultad de Psicología. Universidad de Buenos Aires, 2008. Disponible en: <http://www.psi.uba.ar/academica/ carrerasdegrado/ps>.

La red de apoyo social. Evaluación e intervención con personas adultas mayores. Revista de Psicologia da IMED, v.1, n.1, 2009. Disponible en: <http://www.imed. edu.br/revistapsico $>$.

.; CASTAÑEIRAS, C.; POSADA, M. C. ¿Las fortalezas personales se incrementan en la vejez?: reflexiones acerca del capital psíquico. En: IACUB, R. y cols. Desafíos y logros frente al bien-estar en el envejecimiento. Buenos Aires: Eudeba, 2009.

; POSADA, M. C.; CASTAÑEIRAS, C. El capital psíquico en adultos jóvenes y adultos mayores de la ciudad de Mar del Plata: análisis comparativo de dimensiones e ítems. En: CONGRESO ARGENTINO DE PSIQUIATRÍA, 25. 22 al 25 de abril de 2009. Actas... Mar del Plata.

.; IACUB, R.; SOLIVEREZ, C. y cols. Capital psíquico y bienestar psicológico en adultos jóvenes y adultos mayores residentes en la ciudad de Mar del Plata y Buenos Aires. Proyecto de investigación 2009/2010. Universidad Nacional de Mar del Plata, 2008.

$\therefore$ SABATINI, B. Experiencias de fluidez en adultos y adultos mayores de la ciudad de Mar del Plata. En: JORNADA DE GERIATRÍA Y GERONTOLOGÍA DEL NOA, 5. Trabajo Interdisciplinario con Adultos Mayores: Desafíos y Propuestas. San Miguel de Tucumán 8 de Noviembre de 2008 .

; SOLIVEREZ, C. Análisis dimensional y global del bienestar psicológico en adultos mayores de la ciudad de mar del plata según tipo de hogar. 2009. Disponible en: <http://www.psiquiatria.com/ artículos/psicogeriatria/39939/portalpsiquiatria.com>.

Bienestar psicológico y redes sociales. En: IACUB, R. y cols. Desafíos y logros frente al bienestar en el envejecimiento. Buenos Aires: Eudeba, $2009 b$.

BALTES, P. B.; FREUND, A. M. (2007). El potencial humano como orquestación de la sabiduría y la optimización selectiva con compensaciones. En: ASPINWALL, L. G.; STAUDINGER, U. M. (Eds.). Psicología del potencial humano: cuestiones fundamentales y normas para una Psicología Positiva. Barcelona: Gedisa, 2007. p.45-62.

CARR, A. Psicología Positiva: la ciencia de la felicidad. Barcelona: Paidós Ibérica, 2007.

CARSTENSEN, L. L.; CHARLES, S. T. Emotion in the second half of life. Current Directions in Psychological Science, v.7, p.144-149, 1998.

;_ El envejecimiento humano: ¿por qué incluso las buenas noticias se toman como malas? En: ASPINWALL, L. G.; STAUDINGER, U. M. (Eds.) Psicología del potencial humano: cuestiones fundamentales y normas para una Psicología Positiva. Barcelona: Gedisa, 2007. p. 111- 126.

CARSTENSEN, L.; PASUPATHI, M.; MAYR, U.; NESSELROADE, J. Emotional experience in everyday life across the adult life span. Journal of Personality and Social Psychology, v.79, n.4, 644-655, 2000.

CASUllO, M.; BRENLlA, M. E.; CASTRO SOLANO, A. ; CRUZ, M. S.; GONZALEZ, R.; MAGANTO, C.; MARTÍN, M. ; MARTINEZ, P.; MONTOYA, R.; MOROTE, R. Evaluaciones del Bienestar Psicológico en Iberoamérica. Buenos Aires: Paidós, 2002.

CASUlLO, M. El Capital Psíquico. Aportes de la Psicología Positiva. Psicodebate, v.6, p.59-72, 2006.

CSIKSZENTMIHALYI， M.; CSIKSZENTMIHALYI， I. Experiencia óptima: estudios psicológicos del flujo en la conciencia. Bilbao: Descleé de Brouwer, 1998.

CHICO LIBRÁN, E. Personality dimensions and subjective well- being. The Spanish Journal of Psychology, v.9, n.1, p.38-44, 2006.

DUE, P. HOLSTEIN, B. LUND, R. MODVIG, J.; AVLUND, K. Social relations: network, support and relational strain. 
Social Science \& Medicine, v.48, n.5, p.661-73, 1999.

FERNÁNDEZ BALLESTEROS, R. Luces y sombras en la psicología del potencial humano: el ejemplo de la psicogerontología. En: ASPINWALL, L. G.; STAUDINGER, U. M. (Eds.) Psicología del potencial humano: cuestiones fundamentales y normas para una Psicología Positiva. Barcelona: Gedisa, 2007. p.183- 204.

FRAZIER, C. H.; MINTZ, L. B.; MOBLEY, M. A multidimensional look at religious involvement and psychological well-being among urban elderly african amerians. Journal of Counseling Psychology, v.52, n.4, p.583-590, 2005.

GANCEDO, M. Historia de la Psicología Positiva. Antecedentes, Aportes y Proyecciones. En: CASULLO, M. M. (Comp.). Prácticas en Psicología Positiva. Buenos Aires: Lugar, 2008.

GANEM, M. La Sexualite des seniors. 2002. Disponible en: <http://www.adirs.org $>$.

GARCÍA-VINIEGRAS, C. R.; GONZÁLEZ BENÍTEZ, I. La Categoría Bienestar Psicológico. Su relación con otras categorías sociales. Revista Cubana Medicina General Integral, v.16, n.6, p.586-92, 2000.

IACUB, R. Erótica y vejez. Buenos Aires: Paidós, 2006.

Estéticas de la existencia: ¿la vida es bella en la vejez? Perspectivas en Psicología, v.5, n.2, p.10-17, 2008.

IZAL, M.; MONTORIO, I. Determinantes del bienestar psicológico en la vejez. Revista Argentina de Clínica Psicológica, v.2, p.147-159, 1993.

JANUS, S.; JANUS, C. The Janus report on sexual behavior. Nueva York: John Wiley \& Sons, 1993.

LACEY, H. P.; SMITH, D. M.; UBEL, P. A. Hope I Die Before I Get Old: Mispredicting Happiness Across the Adult Lifespan. Journal of Happiness Studies, v.7, n.2, 167-182, 2006.

LAWTON, M; MOSS, M.; WINTER, L.; HOFFMAN, C. Motivation in later life: personal projects and well-being. Psychology and Aging, v.17, n.4, 539-547, 2002.

LEVIN, J. S.; CHATTERS, L. M. Religion, health, and psychological well-being in older Adults. Journal of Aging and Health, v.10, n.4, p.504-531, 1998.

LIBERALESSO NERI, A. Bienestar subjetivo en la vida adulta y en la vejez: hacia una Psicología Positiva en América Latina. Revista Latinoamericana de Psicología, v.34, n.1-2, p.55-74, 2002.

MASTERS, W.; JONSON, V.; KOLODNY, R. Eros: los mundos de la sexualidad. Barcelona: Grijalbo Mondadori, 1995.

MUCHINIK, E. Hacia una nueva imagen de la vejez. Buenos Aires: Belgrano, 1984.
OKUN, M. A; STOCK, W. A; HARING M. J.; WITTER, R. A. The social activity/subjetive well- being relation. Research on Aging, v.6, n.1, p.45-65, 1984.

POSADA, M. C.; CASTAÑEIRAS, C. E.; ARIAS, C. J. Capital psíquico en población general: diferencias entre varones y mujeres. En: CONGRESO DE PSICOLOGÍA, 2. Facultad de Psicología. Actas... Universidad Nacional de Córdoba, 30 de octubre de 2008. 2008a

POSADA, M. C.; CASTAÑEIRAS, C.; ARIAS, C. (2008b). Dimensiones del capital psíquico en población general. Estudio comparativo por grupos de edad. En: ENCUENTRO IBEROAMERICANO DE PSICOLOGÍA POSITIVA, 3. Universidad de Palermo. Buenos Aires. Actas...

POSADA, M. C.; CASTAÑEIRAS, C. E.; ARIAS, C. J. Relación entre autoconcepto y capital psíquico en adultos de la ciudad de Mar del Plata. En: JORNADAS DE INVESTIGACIÓN Y CUARTO ENCUENTRO DE INVESTIGADORES EN PSICOLOGÍA DEL MERCOSUR, 15. Actas... Facultad de Psicología. Universidad de Buenos Aires. (2008c).

RIVERA LEDESMA, A.; MONTERO LÓPEZ, L. M. Medidas de afrontamiento y espiritualidad en adultos mayores mexicanos. Salud Mental, v.30, n.1, 39-47, 2007.

RYFF, C. D. In the eye of the beholder: views of psychological well-being among middle-aged and older adults. Psychology and Aging, v.4, n.2, p.195-210, 1989.

RYFF, C. D.; SINGER, B. Ironías de la condición humana: bienestar y salud en el camino a la mortalidad. En: ASPINWALL, L. G.; STAUDINGER, U. M. (Eds.) Psicología del potencial humano: cuestiones fundamentals y normas para una Psicología Positiva. Barcelona: Gedisa, 2007. p.111- 126

SABATINI, B.; ARIAS, C. Análisis de las experiencias de fluidez: estudio comparativo en adultos jóvenes y adultos mayores de la ciudad de Mar del Plata. En: CONGRESO INTERNACIONAL DE INVESTIGACIÓN Y PRÁCTICA PROFESIONAL EN PSICOLOGÍA, 1. XVI JORNADAS DE INVESTIGACIÓN Y QUINTO ENCUENTRO DE INVESTIGADORES EN PSICOLOGÍA DEL MERCOSUR, 16. Actas... Facultad de Psicología: UBA. 2009.

SELIGMAN, M. Building human strenght: psychology`s forgotten misión. APA Monitor, v.29, n.1, 1998. Disponible en: <www.apa.org/monitor/jan98/press.html>.

SELIGMAN, M. La auténtica felicidad. Barcelona: Ediciones B., 2003.

SELIGMAN, M.; CSIKSZENTMIHALYI, M. Positive Psychology: an introduction. American Psychologist, v.55, p.5-14, 2002. 
SELIGMAN, M. E.; STEEN, T.; PARK, N.; PETERSON, C. Positive Psychology progress: empirical validation of interventions. American Psychologist, v.60, n.5, p. 410421, 2005.

VERA NORIEGA, J. A; SOTELO QUIÑONES, T. I.; DOMÍNGUEZ GUEDEA, M. I. Bienestar subjetivo, enfrentamiento y redes de apoyo social en adultos mayores. Revista Intercontinental de Psicología y Educación, v.7, n.2, p.57-78, 2005.

VILLAR, F.; TRIADO, C.; SOLÉ, C., OSUNA, M., Bienestar, adaptación y envejecimiento: cuando la estabilidad significa cambio. Revista Multidisciplinar de Gerontología. v.13, n.3, p.152-162, 2003.

YOFFE, L. Religión, espiritualidad y sentido de la vida en los duelos. En: CASUlLO, M. M. (Comp.). Prácticas en Psicología Positiva. Buenos Aires: Lugar, 2008.

WOOD, S. ; KISLEY, M.; BURROWS, L. Looking at the sunny side of life: age-related change in an event-related potential measure of the negativity bias. Psychological Science, v.18, n.9, p.838-843, 2007.

Artigo Submetido: 10/10/2013

Artigo Aprovado: 30/11/2013 